\title{
Beyond Mean-Field Corrections to the Quasiparticle Spectrum of Superfluid Fermi Gases
}

\author{
Senne Van Loon $\odot, "$ Jacques Tempere $\odot$, and Hadrien Kurkjian $\odot$ \\ TQC, Universiteit Antwerpen, Universiteitsplein 1, B-2610 Antwerpen, België
}

(Received 20 August 2019; revised manuscript received 2 December 2019; accepted 21 January 2020; published 21 February 2020)

\begin{abstract}
We investigate the fermionic quasiparticle branch of superfluid Fermi gases in the Bardeen-CooperSchrieffer (BCS) to Bose-Einstein condensation (BEC) crossover and calculate the quasiparticle lifetime and energy shift due to its coupling with the collective mode. The only close-to-resonance process that lowenergy quasiparticles can undergo at zero temperature is the emission of a bosonic excitation from the phononic branch. Close to the minimum of the branch we find that the quasiparticles remain undamped, allowing us to compute corrections to experimentally relevant quantities such as the energy gap, location of the minimum, effective mass, and Landau critical velocity.
\end{abstract}

DOI: 10.1103/PhysRevLett.124.073404

Introduction.-The notion of quasiparticles is an essential tool for the study of interacting many-body systems. The idea is that the many-body problem can be considerably simplified by introducing weakly interacting elementary excitations above a known ground state. It is, however, generally known that fermionic quasiparticles in an interacting Fermi system are only well-defined in a small region around the dispersion minimum, and that elsewhere they acquire a finite lifetime, even at zero temperature [1]. In a superfluid, the fermionic branch, which consists of pair-breaking excitations, obtains a finite energy gap described by BCS theory [2], and the system exhibits a gapless bosonic mode (unlike in superconductors [3]) representing the collective motion of fermion pairs [4]. In this Letter, we identify the coupling of the fermionic quasiparticle to the bosonic collective branch as the mechanism responsible for its finite lifetime away from the dispersion minimum, and compute the corresponding damping rate and energy shift from first principles. This problem is quite similar to the Bose polaron (an impurity in a sea of bosons) [5-7], but a crucial difference is that the fermionic quasiparticle has a rotonlike dispersion $\epsilon_{k}=\Delta+\hbar^{2}\left(k-k_{0}\right)^{2} / 2 m^{*}$, rather than an impurity-like dispersion $\epsilon_{k}=\hbar^{2} k^{2} / 2 m^{*}$.

The appearance of a finite quasiparticle lifetime away from the energy minimum is a many-body phenomenon that occurs in many quantum systems such as normal Fermi liquids [1,8], superconductors [9], or rotonic systems (superfluid Helium [10] or dipolar gases [11]). Using ultracold fermionic atoms [12-18], it can be studied analytically from a first-principle microscopic approach, and compared to experimental observations. Measurements of the quasiparticle spectrum using rf spectroscopy [19] and momentum-resolved rf spectroscopy [20], in particular of the quasiparticle gap [21], are in fact already available. Moreover, using Feshbach resonances to tune the interaction strength [22], experiments can study this effect in the whole range between a weakly interacting BCS-type superfluid and a Bose-Einstein condensate (BEC) of tightly bound dimers, including at unitarity where interactions are resonant.

Yet this problem was somehow overlooked in recent theoretical studies, concentrating rather on computing the equation of state [23-25], the order parameter [26-28], or the bosonic collective mode spectrum [4,29-33]. Pioneering studies have looked at beyond mean-field corrections to the single-particle Green's function [34-36], identifying the coupling to the collective mode as the most important effect [36], but could not extract analytically the corrected eigenenergy and damping rate; moreover, such numerical approaches suffer limitations, predicting in particular a finite lifetime of the quasiparticles at the dispersion minimum. Here, we aim to fill this gap by analytically studying the coupling of the fermionic branch with the bosonic collective mode, modifying its dispersion in the entire BCS-BEC crossover. As expected, we find the quasiparticles to be well defined around the Fermi level, while their lifetime becomes finite away from the energy minimum. The correction is perturbative in both the BCS and the BEC limit, expressing a vanishing damping rate in these limits.

Quasiparticle Hamiltonian.-We study a gas of neutral fermionic atoms of mass $m$ in two different hyperfine states, interacting via an attractive short-range potential, fully characterized, at low energy, by its $s$-wave scattering length $a$. Instead of using the full microscopic Hamiltonian, we describe the weakly excited state of the system in terms of its quasiparticles. Specifically, we use an effective Hamiltonian derived from first principles that describes the fermionic quasiparticles and their coupling to the Anderson-Bogoliubov bosonic collective modes [31] 


$$
\begin{aligned}
\hat{H}_{\mathrm{qp}}= & \sum_{\mathbf{k}, \sigma=\uparrow, \downarrow} \epsilon_{\mathbf{k}} \hat{\gamma}_{\mathbf{k}, \sigma}^{\dagger} \hat{\gamma}_{\mathbf{k}, \sigma}+\sum_{\mathbf{q}} \hbar \omega_{\mathbf{q}} \hat{b}_{\mathbf{q}}^{\dagger} \hat{b}_{\mathbf{q}} \\
& +\frac{1}{\sqrt{V}} \sum_{\mathbf{k}, \mathbf{q}, \sigma}\left(\mathcal{A}_{\mathbf{k}-\mathbf{q}, \mathbf{q}} \hat{b}_{\mathbf{q}}^{\dagger}+\mathcal{A}_{\mathbf{k},-\mathbf{q}} \hat{b}_{-\mathbf{q}}\right) \hat{\gamma}_{\mathbf{k}-\mathbf{q}, \sigma}^{\dagger} \hat{\gamma}_{\mathbf{k}, \sigma} .
\end{aligned}
$$

Here, the first term describes the BCS quasiparticles with creation (annihilation) operators $\hat{\gamma}_{\mathbf{k}, \sigma}^{\dagger}\left(\hat{\gamma}_{\mathbf{k}, \sigma}\right)$ and energy $\epsilon_{\mathbf{k}}=\sqrt{\xi_{\mathbf{k}}^{2}+\Delta^{2}}$, with $\xi_{\mathbf{k}}=\left(\hbar^{2} k^{2} / 2 m\right)-\mu$ the freefermion dispersion relation, $\Delta$ the mean-field gap and $\mu$ the chemical potential. The second term in Eq. (1) represents the free bosonic collective modes, calculated within the RPA or Gaussian pair fluctuations (GPF) approximation $[3,4,24]$, with operators $\hat{b}_{\mathbf{q}}^{\dagger}, \hat{b}_{\mathbf{q}}$; their eigenenergy $\hbar \omega_{\mathbf{q}}$ is the only positive real root of the gaussian fluctuation matrix $\operatorname{det} M\left(\omega_{\mathbf{q}}, \mathbf{q}\right)=0$ [4], with

$M_{ \pm \pm}(\omega, \mathbf{q})=\frac{1}{2 V} \sum_{\mathbf{k}}\left[\frac{\epsilon_{\mathbf{k}_{+}}+\epsilon_{\mathbf{k}_{-}}}{\epsilon_{\mathbf{k}_{+}} \epsilon_{\mathbf{k}_{-}}} \frac{\epsilon_{\mathbf{k}_{+}} \epsilon_{\mathbf{k}_{-}}+\xi_{\mathbf{k}_{+}} \xi_{\mathbf{k}_{-}} \pm \Delta^{2}}{\hbar^{2} \omega^{2}-\left(\epsilon_{\mathbf{k}_{+}}+\epsilon_{\mathbf{k}_{-}}\right)^{2}}+\frac{1}{\epsilon_{\mathbf{k}}}\right]$,

$M_{+-}(\omega, \mathbf{q})=\frac{\hbar \omega}{2 V} \sum_{\mathbf{k}} \frac{1}{\epsilon_{\mathbf{k}_{+}} \epsilon_{\mathbf{k}_{-}}} \frac{\epsilon_{\mathbf{k}_{+}} \xi_{\mathbf{k}_{-}}+\xi_{\mathbf{k}_{+}} \epsilon_{\mathbf{k}_{-}}}{\omega^{2}-\left(\epsilon_{\mathbf{k}_{+}}+\epsilon_{\mathbf{k}_{-}}\right)^{2}}$,

and $\mathbf{k}_{ \pm}=\mathbf{k} \pm \mathbf{q} / 2$. Finally, the second line of Eq. (1) describes the three-body coupling between the fermionic quasiparticles and the collective modes, with coupling amplitude

$$
\mathcal{A}_{\mathbf{k}, \mathbf{q}}=\frac{w_{\mathbf{k q}}^{+} \sqrt{M_{++}\left(\omega_{\mathbf{q}}, \mathbf{q}\right)}+w_{\mathbf{k q}}^{-} \sqrt{M_{--}\left(\omega_{\mathbf{q}}, \mathbf{q}\right)}}{\sqrt{-\left.\frac{2}{\hbar} \frac{\partial}{\partial \omega} \operatorname{det} M(\omega, \mathbf{q})\right|_{\omega=\omega_{\mathbf{q}}}}},
$$

with the weights $w_{\mathbf{k q}}^{ \pm}=U_{\mathbf{k}+\mathbf{q}} V_{\mathbf{k}} \pm U_{\mathbf{k}} V_{\mathbf{k}+\mathbf{q}}$, and $U_{\mathbf{k}}=$ $\sqrt{\left(1+\xi_{\mathbf{k}} / \epsilon_{\mathbf{k}}\right) / 2}, V_{\mathbf{k}}=\sqrt{\left(1-\xi_{\mathbf{k}} / \epsilon_{\mathbf{k}}\right) / 2}$. The effective Hamiltonian (1) describes accurately the emission or absorption of one Anderson-Bogoliubov boson. In principle, it also contains higher order decay processes such as the successive emission of two bosons, but to include those higher order terms consistently one should consider a three-boson coupling Hamiltonian [37], yet unknown in literature on Fermi condensates. We also omit highly offresonant processes such as four-fermion interactions (at $T=0: \gamma^{\dagger} \gamma^{\dagger} \gamma^{\dagger} \gamma$ or $\gamma^{\dagger} \gamma^{\dagger} \gamma^{\dagger} \gamma^{\dagger}$ ) and simultaneous creation of two fermions and a boson $\left(\gamma^{\dagger} \gamma^{\dagger} b^{\dagger}\right)$, that lead to a nonzero contribution to the damping rate only when the quasiparticle energy is at least $3 \Delta$. The process described by $\hat{H}_{\mathrm{qp}}$ is the most relevant damping process at zero temperature and low energy.

Energy corrections.-To study the fermionic branch we compute its Green's function [38], which contains all the information on the quasiparticle. At zero temperature, the collective modes are unoccupied, forbidding the absorption of a boson by the fermionic quasiparticle. In this way, the only second order diagram contributing to the Green's function is the self-energy where the fermion emits and reabsorbs a boson:

$$
G_{\mathbf{k}}(z)=\left(z-\epsilon_{\mathbf{k}}-\frac{1}{V} \sum_{\mathbf{q}} \frac{\left|\mathcal{A}_{\mathbf{k}-\mathbf{q}, \mathbf{q}}\right|^{2}}{z-\epsilon_{\mathbf{k}-\mathbf{q}}-\hbar \omega_{\mathbf{q}}}\right)^{-1} .
$$

As explained at the end of this Letter, one obtains the same quasiparticle Green's function within the formalism of Refs. [34,36] provided one omits all far-off-shell processes. The poles of the Green's function $G_{\mathbf{k}}^{-1}\left(z_{\mathbf{k}}\right)=0$ are the eigenenergies of fermionic quasiparticles dressed by their interactions with the boson bath. When the coupling amplitude $\mathcal{A}$ is small [39], one can replace $z$ by $\epsilon_{\mathbf{k}}+i 0^{+}$in the last term between brackets of Eq. (4), to obtain the energy correction $z_{\mathbf{k}}^{(2)}=E_{\mathbf{k}}^{(2)}-i \hbar \Gamma_{\mathbf{k}} / 2$ to second order perturbation theory:

$$
\begin{aligned}
& E_{\mathbf{k}}^{(2)}=\epsilon_{\mathbf{k}}+\frac{1}{V} \mathcal{P} \sum_{\mathbf{q}} \frac{\left|\mathcal{A}_{\mathbf{k}-\mathbf{q}, \mathbf{q}}\right|^{2}}{\epsilon_{\mathbf{k}}-\epsilon_{\mathbf{k}-\mathbf{q}}-\hbar \omega_{\mathbf{q}}}, \\
& \hbar \Gamma_{\mathbf{k}}=\frac{2 \pi}{V} \sum_{\mathbf{q}}\left|\mathcal{A}_{\mathbf{k}-\mathbf{q}, \mathbf{q}}\right|^{2} \delta\left(\epsilon_{\mathbf{k}}-\epsilon_{\mathbf{k}-\mathbf{q}}-\hbar \omega_{\mathbf{q}}\right) .
\end{aligned}
$$

The resonance condition $\epsilon_{\mathbf{k}}-\epsilon_{\mathbf{k}-\mathbf{q}}-\hbar \omega_{\mathbf{q}}=0$ in (6) is satisfied provided $\epsilon_{\mathbf{k}}$ is inside the boson emission continuum $\left\{\epsilon_{\mathbf{k}-\mathbf{q}}+\hbar \omega_{\mathbf{q}}, \mathbf{q}\right\}$, that is, strictly superior to the threshold energy $\epsilon_{\mathrm{th}}=\min _{\mathbf{q}}\left[\epsilon_{\mathbf{k}-\mathbf{q}}+\hbar \omega_{\mathbf{q}}\right]$, which is also the lower edge of the branch cut of $G_{\mathbf{k}}(z)$.

Close to the minimum $k_{0}$ of the unperturbed fermionic branch, the group velocity of the quasiparticle is smaller than the sound velocity $c$ of the collective mode $\left|\partial \epsilon_{k} / \partial k\right|<\hbar c$. For these values of $k$, the minimum $\min _{u}\left[\epsilon_{\mathbf{k}-\mathbf{q}}+\hbar \omega_{\mathbf{q}}\right]$ over the scattering angle $u=\mathbf{k} \cdot \mathbf{q} / k q$ is a strictly increasing function of $q$, starting from its lowest value $\epsilon_{\mathbf{k}}$ in $q=0$, such that $\epsilon_{\mathrm{th}}=\epsilon_{\mathbf{k}}$ and the decay by emission of collective modes is energetically forbidden $[31,36]$. The perturbative damping rate is zero $\left(\Gamma_{\mathbf{k}}=0\right)$ and we find correspondingly a real pole of $G_{\mathbf{k}}$, indicating that the quasiparticles, despite their renormalization by the bosonic bath, remain well defined close to the minimum of their dispersion.

When the group velocity $\left|\partial \epsilon_{k} / \partial k\right|$ becomes larger than $\hbar c$ (which can happen in both the increasing $\left[k>k_{0}\right]$ and decreasing parts $\left[k<k_{0}\right]$ of the BCS branch), the unperturbed energy $\epsilon_{\mathbf{k}}$ becomes greater than $\epsilon_{\mathrm{th}}$ and the resonance condition of Eq. (6) can be satisfied. Although the perturbative damping rate $\Gamma_{\mathbf{k}}$ becomes nonzero, the selfconsistent solution $z_{\mathbf{k}}$ below $\epsilon_{\text {th }}$ remains real for larger values of $\left|k-k_{0}\right|$. Eventually, $z_{\mathbf{k}}$ also enters the continuum and becomes imaginary, resulting in a broadened peak in the spectral function $\varepsilon \mapsto \operatorname{Im}\left[G_{\mathbf{k}}\left(\varepsilon+i 0^{+}\right)\right]$.

In Fig. 1, we illustrate this by plotting $\varepsilon \mapsto \operatorname{Im} G_{\mathbf{k}}\left(\varepsilon+i 0^{+}\right)$ at unitarity $\left(1 / k_{F} a=0\right.$, where $k_{F}$ is the Fermi wave number) as a function of $\varepsilon$ and $k$. We superimpose the eigenenergy $z_{\mathbf{k}}$ obtained by self-consistently solving for the real pole of the Green's function below $\epsilon_{\mathrm{th}}$, and the 


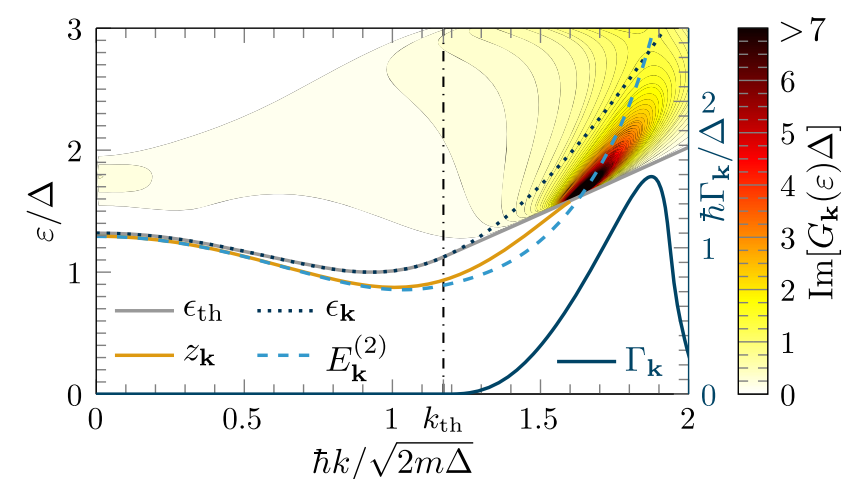

FIG. 1. The imaginary part of the quasiparticle Green's function (4) is shown as a function of the wavenumber $k$ and energy $\varepsilon$ in units of $\Delta$. It is nonzero only above the threshold energy $\epsilon_{\text {th }}$ (solid gray line). Black dotted line: mean field energy $\epsilon_{\mathbf{k}}$, blue dashed line: perturbative eigenfrequency $E_{\mathbf{k}}^{(2)}$, blue solid line: perturbative damping rate $\Gamma_{\mathbf{k}}$. The latter is nonzero only for $k>k_{\mathrm{th}} \simeq 1.17 \sqrt{2 m \Delta} / \hbar$ (vertical dashed dotted line) with $\left|\partial \epsilon_{\mathbf{k}} / \partial k\right|_{k=k_{\mathrm{th}}}=\hbar c$. Below the threshold energy, a self-consistent energy $z_{\mathbf{k}}$ can be found until $k \simeq 1.62 \sqrt{2 m \Delta} / \hbar>k_{\text {th }}$ (orange solid line). It remains relatively close to the perturbative energy.

perturbative result $E_{\mathbf{k}}^{(2)}$ of Eq. (5), which remains fairly close to $z_{\mathbf{k}}$ everywhere. Once the self-consistent solution hits the continuum, the exact resonance of $G_{\mathbf{k}}$ turns into a broadened peak at energies $\varepsilon>\epsilon_{\mathrm{th}}$. The perturbative damping $\Gamma_{\mathbf{k}}$ becomes nonzero at $k=k_{\text {th }}$ with $\left|\partial \epsilon_{\mathbf{k}} / \partial k\right|_{k=k_{\text {th }}}=\hbar c$. It is then highly peaked when the energy $\epsilon_{\mathbf{k}}$ is around $3 \Delta$. This could suggest that the $1 \rightarrow 3$ fermionic processes that we excluded from our Hamiltonian $\hat{H}_{\mathrm{qp}}$ in Eq. (1) become important above $3 \Delta$. At higher wave numbers, $E_{\mathbf{k}}^{(2)}$ approaches the mean field result (as does the maximum of $\left.\operatorname{Im}\left[G_{\mathbf{k}}\left(\varepsilon+i 0^{+}\right)\right]\right)$. This is not a surprise since the coupling $\mathcal{A}$ is comparatively small in the limit $k \rightarrow \infty$.

$B C S$ and BEC limits. - The perturbative treatment of Eqs. (5) and (6) (already close to the self-consistent solution at unitarity) gives asymptotically the exact solution of $G_{\mathbf{k}}^{-1}=0$ in the BCS and BEC limits $(\Delta /|\mu| \rightarrow 0)$. In the BCS limit, the bosonic wave numbers should be expressed in units of $\Delta / \hbar c$, such that the dispersion $\hbar \omega_{\mathbf{q}} / \Delta$ becomes a universal function of $\tilde{\mathbf{q}}=\hbar c \mathbf{q} / \Delta$ [40]. The energy correction $\left|z_{\mathbf{k}}-\epsilon_{\mathbf{k}}\right|$ is of order $\Delta^{2} / \mu^{2}$ :

$\frac{z_{\mathbf{k}}^{(2)}-\epsilon_{\mathbf{k}}}{\Delta}=\frac{\Delta^{2}}{\mu^{2}} \frac{1}{V} \sum_{\tilde{\mathbf{q}}} \frac{\left(w_{\mathbf{k q}}^{-}\right)^{2}}{K(\tilde{\mathbf{q}})} \frac{\Delta}{\epsilon_{\mathbf{k}}-\epsilon_{\mathbf{k}-\mathbf{q}}-\hbar \omega_{\mathbf{q}}+i 0^{+}}$,

with $K(\tilde{\mathbf{q}})=-32 \hbar \Delta /\left(3 \sqrt{3} m k_{F}\right) \partial M_{++} /\left.\partial \omega\right|_{\omega=\omega_{\mathbf{q}}}$ a universal function of $\tilde{\mathbf{q}}$. In the BEC limit, the energy should be scaled to the chemical potential $|\mu|$ and analytic results are available for the collective mode dispersion [4] at arbitrary momentum $q$. Consequently, the energy $E_{\mathbf{k}}^{(2)}$ and damping rate $\Gamma_{\mathbf{k}}$ of Eqs. (5) and (6) can be computed analytically, resulting in
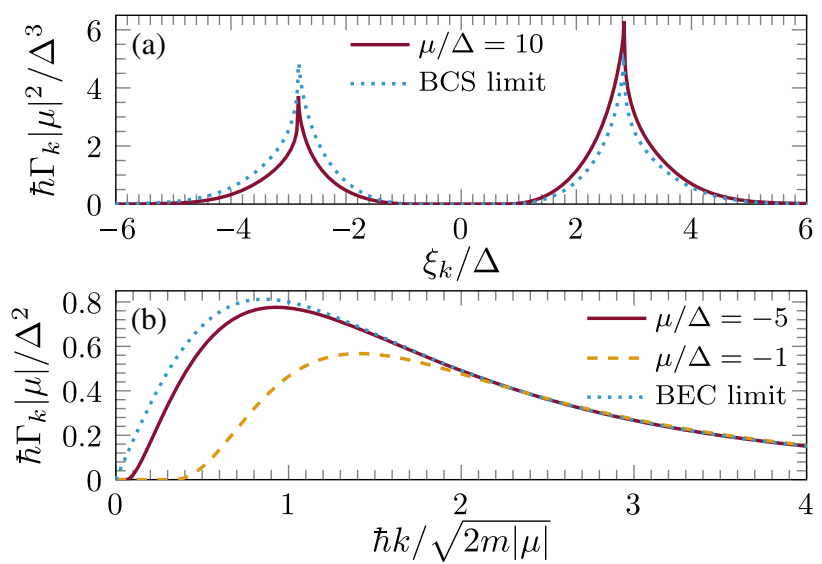

FIG. 2. Perturbative damping rate $\Gamma_{\mathbf{k}}$ (6) for different interaction regimes. (a) The damping rate as a function of $\xi_{k}=$ $\hbar^{2} k^{2} / 2 m-\mu$ at $\mu / \Delta=10$ (full red line) is rescaled to compare with the universal behavior in the BCS limit (dotted blue line), where the damping rate vanishes as $\Delta^{2} / \mu^{2}$, according to Eq. (7). (b) Damping rate in the BEC regime as a function of $\hbar k / \sqrt{2 m|\mu|}$ for $\mu / \Delta=-5$ (full red line), and $\mu / \Delta=-1$ (dashed orange line) and $\mu / \Delta=-\infty$ [dotted blue line, see Eq. (8)].

$$
\frac{z_{\mathbf{k}}^{(2)}-\epsilon_{\mathbf{k}}}{|\mu|}=\frac{\Delta^{2}}{|\mu|^{2}} \frac{2 \tilde{k}^{2}-6-8 i \tilde{k}}{\tilde{k}^{4}+10 \tilde{k}^{2}+9},
$$

with $\tilde{k}=\hbar k / \sqrt{2 m|\mu|}$. The quasiparticle lifetime thus diverges like $\mu^{2} / \Delta^{2}$.

In Fig. 2 we examine the perturbative damping rate $\Gamma_{\mathbf{k}}$ of Eq. (6) in the BCS and BEC limits. In the BCS limit, the undamped region lies around $k_{0} \approx k_{F}$ and its width in units of $k_{F}$ tends to zero like $\Delta / \varepsilon_{F}$. Outside this region, a highly peaked behavior can be observed, which, as in the unitary case, occurs when the energy is close to $3 \Delta$. In the BEC limit, the undamped region lies around $k_{0}=0$, while the threshold wave number vanishes as $\hbar k_{\mathrm{th}} / \sqrt{2 m|\mu|}=\Delta / 4|\mu|$. As a function of $k$, the damping rate smoothens, and exhibits a $1 / k^{3}$ tail at high $k$.

Quadratic dispersion near the minimum.-To analyze the characteristics of the corrected fermionic energy branch, we fit a quadratic dispersion,

$$
\epsilon_{k}^{\mathrm{fit}}=\epsilon^{*}+\frac{\hbar^{2}\left(k-k_{m}^{*}\right)^{2}}{2 m^{*}},
$$

to the minimum of the energy. This allows us to extract the most interesting features of the energy correction, in the region where the description in terms of quasiparticles is certainly accurate. Concretely, these parameters represent the effective energy gap $\epsilon^{*}$, the location of the minimum of the branch $k_{m}^{*}$ and the effective mass $m^{*}$. In Fig. 3 we present these fitting parameters in the BCS-BEC crossover for both the self-consistent energy solution and the perturbative energy correction, comparing with the meanfield version of Eq. (9) obtained by expanding the BCS 


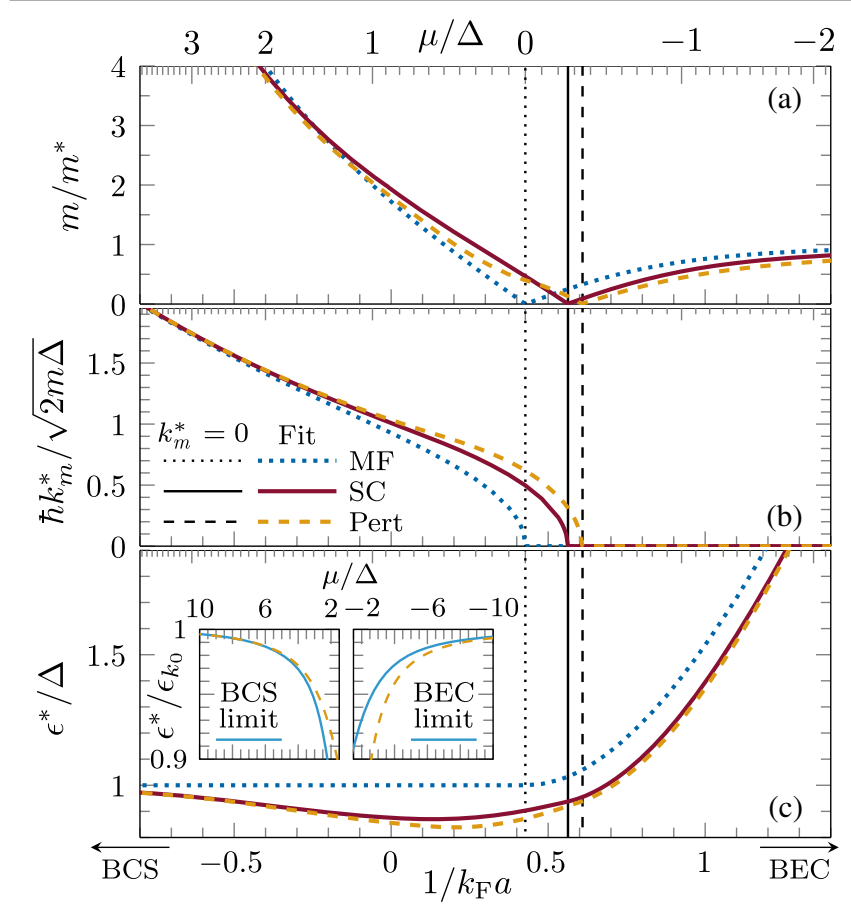

FIG. 3. Minimum of the corrected fermionic energy branch fitted to a quadratic dispersion. (a) Dimensionless inverse effective mass $m / m^{*}$, (b) location of the minimum $\hbar k_{m}^{*} / \sqrt{2 m \Delta}$, (c) energy gap $\epsilon^{*} / \Delta$, shown in function of the inverse scattering length $1 / k_{F} a$ (bottom $x$ axis) and $\mu / \Delta$ (top $x$ axis). Full curves: fitting parameters to the self-consistent (SC) energy solution $z_{\mathbf{k}}$. Dashed lines: perturbative (Pert) results. Dotted lines: mean-field (MF) results. The vertical black lines specify the critical values of the interaction after which the minimum of the energy is located at $k=0$, in their respective line styles. The BCS and BEC asymptotes are drawn for the energy gap in an inset.

energy $\epsilon_{\mathbf{k}}$ around its minimum. All parameters tend to the mean-field result in the BCS and BEC limits, confirming that the energy correction is perturbative for $\Delta /|\mu| \rightarrow 0$. Additionally, the differences between the self-consistent and perturbative results are never substantial, although perturbation theory somewhat overestimates the correction. The interaction with the bosonic collective mode lowers the fermionic energy gap $\epsilon^{*}$, which is to be expected as it is generally known that the mean-field theory exaggerates the gap. At unitarity, we find $\epsilon^{*} \simeq 0.88 \Delta \simeq 0.41 \epsilon_{F}$ (using the GPF equation of state [24]), close to the experimental result $\Delta=0.44 \epsilon_{F}$ [21], and $k_{m}^{*} \simeq 1.01 \sqrt{2 m \Delta} / \hbar \simeq 0.69 k_{F}$. Furthermore, the location $k_{m}^{*}$ of the energy minimum reaches 0 at some critical value $\mu / \Delta \simeq-0.26$, depicted by a vertical line on Fig. 3, corresponding to $1 / k_{F} a \simeq 0.56$. The fact that this happens while the chemical potential is already negative has been theoretically predicted before [36].

Critical velocity.-Another interesting characterization of the fermionic branch is its Landau critical velocity, which determines the maximal velocity for frictionless flow at $T=0$ in a superfluid. As there are two branches of elementary excitations in a superfluid Fermi gas, the critical velocity will be given by the smallest of the two velocities $v_{c}=\min \left[v_{f}, c\right]$, with $v_{f}=\min _{\mathbf{k}_{1}, \mathbf{k}_{2}}\left(\varepsilon_{\mathbf{k}_{1}}+\varepsilon_{\mathbf{k}_{2}}\right) / \hbar\left|\mathbf{k}_{1}+\mathbf{k}_{2}\right|$ the fermionic critical velocity [41]. In the BCS limit the critical velocity is reached for $k$ close to $k_{F}$ and we can use the effective quadratic dispersion (9) near the minimum to compute it. This yields $m^{*} v_{f}^{\text {quad }}=\sqrt{2 m^{*} \epsilon^{*}+\left(\hbar k_{m}^{*}\right)^{2}}-\hbar k_{m}^{*}$, from which we can extract the first deviation to the mean-field velocity $\left(v_{f} / v_{f}^{\mathrm{MF}}\right)=\left(\epsilon^{*} / \Delta\right)+O(\Delta / \mu)^{3} \simeq$ $1-0.5(\Delta / \mu)^{2}+O(\Delta / \mu)^{3}$.

Quasiparticle Green's function.-Finally, we show that the quasiparticle Green's function Eq. (4) can be rederived in a more general microscopic formalism $[34,36,42]$ whose starting point are the particle, hole, and anomalous Green's functions. The lowest order self-energy correcting the mean-field particle-hole Green's function,

$$
\begin{aligned}
\mathcal{G}_{0}(z, \mathbf{k})= & -\frac{1}{z+\epsilon_{\mathbf{k}}}\left(\begin{array}{cc}
V_{\mathbf{k}}^{2} & U_{\mathbf{k}} V_{\mathbf{k}} \\
U_{\mathbf{k}} V_{\mathbf{k}} & U_{\mathbf{k}}^{2}
\end{array}\right) \\
& +\frac{1}{z-\epsilon_{\mathbf{k}}}\left(\begin{array}{cc}
-U_{\mathbf{k}}^{2} & U_{\mathbf{k}} V_{\mathbf{k}} \\
U_{\mathbf{k}} V_{\mathbf{k}} & -V_{\mathbf{k}}^{2}
\end{array}\right),
\end{aligned}
$$

was derived in Ref. [34]: $\mathcal{G}^{-1}=\mathcal{G}_{0}^{-1}-\Sigma$, with

$$
\begin{aligned}
& \Sigma_{\alpha \alpha^{\prime}}(z, \mathbf{k}) \\
& \quad=\frac{1}{V} \sum_{\mathbf{q}} \int_{-i \infty}^{+i \infty} \frac{d z_{q}}{2 \pi i} \mathcal{G}_{0, \alpha^{\prime} \alpha}\left(z_{q}-z, \mathbf{q}-\mathbf{k}\right) \tilde{M}_{\alpha \alpha^{\prime}}^{-1}\left(z_{q}, \mathbf{q}\right) .
\end{aligned}
$$

Here, $\alpha, \alpha^{\prime}=1$ or 2 and $\tilde{M}$ is a unitary transform of $M$ defined in Eq. (2)

$\tilde{M}=-\frac{1}{2}\left(\begin{array}{cc}M_{++}+M_{--}+2 M_{+-} & M_{++}-M_{--} \\ M_{++}-M_{--} & M_{++}+M_{--}-2 M_{+-}\end{array}\right)$.

This self-energy contains corrections from four-fermion processes (the lowest order processes allowed for fermions), as can be seen by expanding $\tilde{M}^{-1}$ in powers of the coupling constant $g_{0}=-\left(\sum_{\mathbf{k}} 1 / 2 \epsilon_{k}\right)^{-1}$. To correctly describe the bosonic spectrum, two of the intermediate fermions are propagated using the ladder-resummed pair propagator $\tilde{M}^{-1}$, see Fig. 7 in Ref. [36]. To obtain analytic results, and avoid spurious effects (such as the appearance of a nonzero imaginary part of $\Delta$ at $T=0$ ), we have dropped the self-consistent treatment of Ref. [36] and used in Eq. (11) the BCS and RPA expressions for $\mathcal{G}_{0}$ and $\tilde{M}^{-1}$. At $T=0, \tilde{M}^{-1}$ has two real poles $\pm \hbar \omega_{\mathbf{q}}$, and two gapped branch cuts. The Matsubara frequency integral in Eq. (11) can then be performed by closing the contour on the real axis, (the most straightforward way is to avoid the poles of $\mathcal{G}_{0}$ ). Neglecting the far-off-shell contributions of the branch cuts of $\tilde{M}^{-1}$, and performing the orthogonal transform to the quasiparticle basis $\tilde{\mathcal{G}}^{-1}=P \mathcal{G}^{-1} P^{\dagger}$ with 


$$
P=\left(\begin{array}{cc}
U_{\mathbf{k}} & -V_{\mathbf{k}} \\
V_{\mathbf{k}} & U_{\mathbf{k}}
\end{array}\right)
$$

we obtain the corrected Green's function

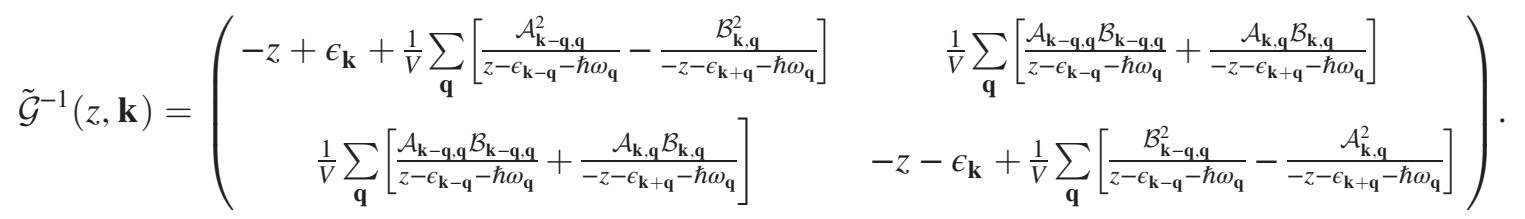

Introducing the weights $W_{\mathbf{k q}}^{ \pm}=U_{\mathbf{k}+\mathbf{q}} U_{\mathbf{k}} \pm V_{\mathbf{k}} V_{\mathbf{k}+\mathbf{q}}$, the amplitude $\mathcal{B}$ is given by

$$
\mathcal{B}_{\mathbf{k}, \mathbf{q}}=\frac{W_{\mathbf{k q}}^{+} \sqrt{M_{--}\left(\omega_{\mathbf{q}}, \mathbf{q}\right)}-W_{\mathbf{k q}}^{-} \sqrt{M_{++}\left(\omega_{\mathbf{q}}, \mathbf{q}\right)}}{\sqrt{-\left.\frac{2}{\hbar} \frac{\partial}{\partial \omega} \operatorname{det} M(\omega, \mathbf{q})\right|_{\omega=\omega_{\mathbf{q}}}}},
$$

while $\mathcal{A}_{\mathbf{k}, \mathbf{q}}$ coincides with Eq. (3). The quasiparticle energy is given by the poles of $\tilde{\mathcal{G}}(z)$, hence the roots of $\operatorname{det} \tilde{\mathcal{G}}^{-1}(z)$. Note the property $\operatorname{det} \tilde{\mathcal{G}}^{-1}(-z)=\operatorname{det} \tilde{\mathcal{G}}^{-1}(z)$, ensuring that the quasiparticle and quasihole energies are simply opposite. To be consistent with the omission of the branch cut contributions, we drop in Eq. (14) the terms corresponding to the far-off-shell processes $\hat{\gamma}_{-\mathbf{k}-\mathbf{q}, \sigma}^{\dagger} \hat{\gamma}_{\mathbf{k}, \sigma}^{\dagger} \hat{b}_{\mathbf{q}}^{\dagger}$, and restrict to first order in $\Sigma$ (which amounts to setting $\mathcal{B}=0$ ). Then $\operatorname{det} \tilde{\mathcal{G}}^{-1}(z)$ is simply the product of the inverse quasiparticle $\tilde{\mathcal{G}}_{11}(z)=-G_{\mathbf{k}}(z)+\mathcal{O}\left(\|\Sigma\|^{2}\right)$ and quasihole $\tilde{\mathcal{G}}_{22}(z)=$ $G_{\mathbf{k}}(-z)+\mathcal{O}\left(\|\Sigma\|^{2}\right)$ Green's functions

$$
\operatorname{det} \mathcal{G}^{-1}=-G_{\mathbf{k}}^{-1}(z) G_{\mathbf{k}}^{-1}(-z)+\mathcal{O}\left(\|\Sigma\|^{2}\right) .
$$

This finally explains the use of Eq. (4) to study the perturbed quasiparticle spectrum.

Conclusion.-We have corrected the fermionic quasiparticle branch by including its interaction with the bosonic collective mode. At low energy, this is the only relevant decay channel, thus giving the sole contribution to the damping of the single-particle excitations. We have computed this damping rate in the entire BCS-BEC crossover, and found real poles of the corrected Green's function close to the minimum of the branch, indicating well-defined quasiparticles.

The boson-emission process we have studied occurs in systems where rotons are present, such as superfluid Helium [10] and dipolar Bose gases [11], and should be responsible at $T=0$ for both a shift of the roton gap and a finite lifetime away from the roton minimum. It occurs also in normal Fermi liquids [1,8] when the quasiparticles (in this case gapless) are coupled to the zero sound branch. In superconductors, the Bogoliubov-Anderson branch is gapped due to Coulomb interactions, but the quasiparticle can emit crystal phonons [9], a process quite similar to the one we have studied. Our technique could also be useful to describe quasiparticle damping in nuclear or neutron matter $[43,44]$.

This research was supported by the Bijzonder Onderzoeksfunds (BOF) of the University of Antwerp, the Fonds Wettenschappelijk Onderzoek Vlaanderen, Project No. G.0429.15.N, and the European Union's Horizon 2020 research and innovation program under the Marie Skłodowska-Curie Grant No. 665501.

"Senne.VanLoon@UAntwerpen.be

[1] P. Nozières, Theory of Interacting Fermi Systems (W.A. Benjamin, New York, 1964).

[2] J. Bardeen, L. N. Cooper, and J. R. Schrieffer, Theory of superconductivity, Phys. Rev. 108, 1175 (1957).

[3] P. W. Anderson, Random-phase approximation in the theory of superconductivity, Phys. Rev. 112, 1900 (1958).

[4] R. Combescot, M. Yu. Kagan, and S. Stringari, Collective mode of homogeneous superfluid Fermi gases in the BECBCS crossover, Phys. Rev. A 74, 042717 (2006).

[5] L. D. Landau, Electron motion in crystal lattices, Phys. Z. 3, 664 (1933).

[6] O. I. Utesov, M. I. Baglay, and S. V. Andreev, Effective interactions in a quantum Bose-Bose mixture, Phys. Rev. A 97, 053617 (2018).

[7] S. Van Loon, W. Casteels, and J. Tempere, Ground-state properties of interacting Bose polarons, Phys. Rev. A 98, 063631 (2018).

[8] H. Godfrin, M. Meschke, H.-J. Lauter, A. Sultan, H. M. Böhm, E. Krotscheck, and M. Panholzer, Observation of a roton collective mode in a two-dimensional Fermi liquid, Nature (London) 483, 576 (2012).

[9] S. B. Kaplan, C. C. Chi, D. N. Langenberg, J. J. Chang, S. Jafarey, and D. J. Scalapino, Quasiparticle and phonon lifetimes in superconductors, Phys. Rev. B 14, 4854 (1976).

[10] B. Fåk, T. Keller, M. E. Zhitomirsky, and A. L. Chernyshev, Roton-Phonon Interactions in Superfluid ${ }^{4} \mathrm{He}$, Phys. Rev. Lett. 109, 155305 (2012).

[11] L. Chomaz, R. M. W. van Bijnen, D. Petter, G. Faraoni, S. Baier, J. H. Becher, M. J. Mark, F. Wächtler, L. Santos, and F. Ferlaino, Observation of roton mode population in a dipolar quantum gas, Nat. Phys. 14, 442 (2018). 
[12] I. Bloch, J. Dalibard, and W. Zwerger, Many-body physics with ultracold gases, Rev. Mod. Phys. 80, 885 (2008).

[13] W. Zwerger, The BCS-BEC Crossover and the Unitary Fermi Gas (Springer-Verlag, Berlin, Heidelberg, 2012), Vol. 836.

[14] M. Greiner, C. A. Regal, and D. S. Jin, Emergence of a molecular Bose-Einstein condensate from a Fermi gas, Nature (London) 426, 537 (2003).

[15] J. Joseph, B. Clancy, L. Luo, J. Kinast, A. Turlapov, and J. E. Thomas, Measurement of Sound Velocity in a Fermi Gas near a Feshbach Resonance, Phys. Rev. Lett. 98, 170401 (2007).

[16] W. Ketterle and M. W. Zwierlein, Making, probing and understanding- ultracold Fermi gases, in Proceedings of the International School of Physics Enrico Fermi, Course CLXIV, edited by M Inguscio, W Ketterle, and C Salomon (IOS Press, Amsterdam, 2008).

[17] L. A. Sidorenkov, M. K. Tey, R. Grimm, Y.-H. Hou, L. Pitaevskii, and S. Stringari, Second sound and the superfluid fraction in a Fermi gas with resonant interactions, Nature (London) 498, 78 (2013).

[18] S. Hoinka, P. Dyke, M. G. Lingham, J. J. Kinnunen, G. M. Bruun, and C. J. Vale, Goldstone mode and pair-breaking excitations in atomic Fermi superfluids, Nat. Phys. 13, 943 (2017).

[19] S. Gupta, Z. Hadzibabic, M. W. Zwierlein, C. A. Stan, K. Dieckmann, C. H. Schunck, E. G. M. van Kempen, B. J. Verhaar, and W. Ketterle, Radio-frequency spectroscopy of ultracold fermions, Science 300, 1723 (2003).

[20] J. T. Stewart, J. P. Gaebler, and D. S. Jin, Using photoemission spectroscopy to probe a strongly interacting Fermi gas, Nature (London) 454, 744 (2008).

[21] A. Schirotzek, Y.-i. Shin, C. H. Schunck, and W. Ketterle, Determination of the Superfluid Gap in Atomic Fermi Gases by Quasiparticle Spectroscopy, Phys. Rev. Lett. 101, 140403 (2008).

[22] M. Bartenstein, A. Altmeyer, S. Riedl, S. Jochim, C. Chin, J. Hecker Denschlag, and R. Grimm, Crossover from a Molecular Bose-Einstein Condensate to a Degenerate Fermi Gas, Phys. Rev. Lett. 92, 120401 (2004).

[23] H. Hu, X.-J. Liu, and P. D. Drummond, Equation of state of a superfluid Fermi gas in the BCS-BEC crossover, Europhys. Lett. 74, 574 (2006).

[24] R. B. Diener, R. Sensarma, and M. Randeria, Quantum fluctuations in the superfluid state of the BCS-BEC crossover, Phys. Rev. A 77, 023626 (2008).

[25] G. E. Astrakharchik, J. Boronat, J. Casulleras, and S. Giorgini, Equation of State of a Fermi Gas in the BECBCS Crossover: A Quantum Monte Carlo Study, Phys. Rev. Lett. 93, 200404 (2004).

[26] L. P. Gor'kov and T. K. Melik-Barkhudarov, Contribution to the theory of superfluidity in an imperfect Fermi gas, ZhETF 40, 1452 (1961)[Sov. Phys. JETP 13, 1018 (1961)].

[27] P Pieri, L Pisani, and G Calvanese Strinati, BCS-BEC crossover at finite temperature in the broken-symmetry phase, Phys. Rev. B 70, 094508 (2004).

[28] L Pisani, P Pieri, and G Calvanese Strinati, Gap equation with pairing correlations beyond the mean-field approximation and its equivalence to a Hugenholtz-Pines condition for fermion pairs, Phys. Rev. B 98, 104507 (2018).

[29] V. A. Andrianov and V. N. Popov, Gidrodinamieskoe dejstvie i Boze-spektr sverhtekuih Fermi-sistem, Teor. Mat. Fiz. 28, 341 (1976) [Hydrodynamic action and Bose spectrum of superfluid Fermi systems, Theor. Math. Phys. 28, 829 (1976)].

[30] H. Kurkjian, Y. Castin, and A. Sinatra, Three-phonon and four-phonon interaction processes in a pair-condensed Fermi gas, Ann. Phys. (N.Y.) 529, 1600352 (2017).

[31] H. Kurkjian and J. Tempere, Absorption and emission of a collective excitation by a fermionic quasiparticle in a Fermi superfluid, New J. Phys. 19, 113045 (2017).

[32] H. Kurkjian, S. N. Klimin, J. Tempere, and Y. Castin, PairBreaking Collective Branch in BCS Superconductors and Superfluid Fermi Gases, Phys. Rev. Lett. 122, 093403 (2019).

[33] S. N. Klimin, J. Tempere, and H. Kurkjian, Phononic collective excitations in superfluid Fermi gases at nonzero temperatures, Phys. Rev. A 100, 063634 (2019).

[34] R. Haussmann, Crossover from BCS superconductivity to Bose-Einstein condensation: A self-consistent theory, Z. Phys. B Condens. Matter 91, 291 (1993).

[35] N. Lerch, L. Bartosch, and P. Kopietz, Absence of Fermionic Quasiparticles in the Superfluid State of the Attractive Fermi Gas, Phys. Rev. Lett. 100, 050403 (2008).

[36] R. Haussmann, M. Punk, and W. Zwerger, Spectral functions and rf response of ultracold fermionic atoms, Phys. Rev. A 80, 063612 (2009).

[37] Y. Castin, A. Sinatra, and H. Kurkjian, Landau PhononRoton Theory Revisited for Superfluid ${ }^{4} \mathrm{He}$ and Fermi Gases, Phys. Rev. Lett. 119, 260402 (2017); Erratum, Phys. Rev. Lett. 123, 239904 (2019).

[38] C. Cohen-Tannoudji, J. Dupont-Roc, and G. Grynberg, Chap. III. Étude non perturbative des amplitudes de transition, Processus d'interaction entre photons et atomes (InterEditions et Éditions du CNRS, Paris, 1988).

[39] At strong coupling there is no small parameter guaranteeing the smallness of the coupling amplitude. However, the correction to the eigenenergy never exceeds $15 \%$, see, e.g., Fig 3(c), and the self-consistent result remains close to the perturbative one. This suggests that higher order decay processes where two or more bosons are emitted would lead to even smaller corrections.

[40] H. Kurkjian, Y. Castin, and A. Sinatra, Concavity of the collective excitation branch of a Fermi gas in the BEC-BCS crossover, Phys. Rev. A 93, 013623 (2016).

[41] Y. Castin, I. Ferrier-Barbut, and C. Salomon, La vitesse critique de Landau d'une particule dans un superfluide de fermions, C.R. Phys. 16, 241 (2015).

[42] M. Pini, P. Pieri, and G. Calvanese Strinati, Fermi gas throughout the BCS-BEC crossover: Comparative study of $t$-matrix approaches with various degrees of selfconsistency, Phys. Rev. B 99, 094502 (2019).

[43] H.-J. Schulze, A. Polls, and A. Ramos, Pairing with polarization effects in low-density neutron matter, Phys. Rev. C 63, 044310 (2001).

[44] L. G. Cao, U. Lombardo, and P. Schuck, Screening effects in superfluid nuclear and neutron matter within Brueckner theory, Phys. Rev. C 74, 064301 (2006). 